\title{
ON EXTREMAL BINARY DOUBLY-EVEN SELF-DUAL CODES OF LENGTH $88^{*}$
}

\author{
Radinka Yorgova, Nuray At
}

\begin{abstract}
In this paper we present 35 new extremal binary self-dual doubly-even codes of length 88 . Their inequivalence is established by invariants. Moreover, a construction of a binary self-dual [88, 44,16] code, having an automorphism of order 21 , is given.
\end{abstract}

1. Introduction. Binary self-dual codes are an interesting class of codes for several reasons. These codes include the extended [8,4,4] Hamming code, the extended binary Golay code and the extended binary quadratic residue codes. Many of the self-dual codes are related to block designs, graphs, lattices and other combinatorial structures.

All binary self-dual codes of Type II of length up to 32 and all of Type I of length up to 34 are classified and given in [1], [2], [11], [12] and [15]. It is known that with increasing length the number of self-dual codes grows very fast. For example, there are 85 inequivalent self-dual codes of Type II of length 32 and

ACM Computing Classification System (1998): E.4, H.1.1.

Key words: Automorphisms, self-dual codes.

${ }^{*}$ This work was partly supported by the Norwegian Government Scholarship. 
at least 17000 of length 40 . Therefore, the question of classifying all self-dual codes of a given length loses interest for increasing values of $n$.

In this work we study extremal binary doubly-even self-dual codes of length 88 having an automorphism of order 21 . The greatest length of an extremal doubly-even self-dual code with minimum distance 16 is 88 . The first example of such a code is given in [9, p. 633]. The next 33 codes are presented in [6]. A construction of a self-dual $[88,44,16]$ code having an automorphism of order 5 is given in [5] and 36 new codes are listed. Here we construct 35 new binary $[88,44,16]$ doubly-even self-dual codes. These codes and the previously known 70 codes are inequivalent.

A binary $[n, k]$ code $\mathcal{C}$ is a $k$-dimensional vector subspace of $\mathbb{F}_{2}^{n}$, where $\mathbb{F}_{2}$ is the field of two elements. The weight of a vector is the number of its nonzero coordinates. An $[n, k, d]$ code is an $[n, k]$ code with minimum weight $d$. A code $\mathcal{C}$ is self-dual if $\mathcal{C}=\mathcal{C}^{\perp}$ where $\mathcal{C}^{\perp}$ is the dual code of $\mathcal{C}$ under the standard inner product. A self-dual code $\mathcal{C}$ is doubly-even if all codewords of $\mathcal{C}$ have weight divisible by four, and singly-even if there is at least one codeword of weight $\equiv 2$ (mod 4). Self-dual doubly-even codes exist only when $n$ is a multiple of eight. It is known [13] that for a self-dual $[n, n / 2, d]$ code:

$$
d \leq 4\left[\frac{n}{24}\right]+4, \text { if } n \not \equiv 22(\bmod 24),
$$

and

$$
d \leq 4\left[\frac{n}{24}\right]+6, \text { if } n \equiv 22(\bmod 24) .
$$

If $n$ is a multiple of 24, then any code reaching limit (1) must be doubly-even.

Self-dual codes which reach these bounds are called extremal.

The weight enumerator of a $[n, k]$ code is the polynomial $\sum_{i=1}^{n} A_{i} y^{i}$, where $A_{i}$ is the number of the codewords of weight $i$. The weight enumerator of extremal doubly-even self-dual codes of a given length is uniquely determined [4]. Two binary codes are equivalent if one can be obtained from the other by a permutation of the coordinates. A permutation $\sigma$ of $n$ elements is an automorphism of a code $\mathcal{C}$ if $\mathcal{C}$ coincides with its image $\sigma(\mathcal{C})$. The set of all automorphisms of a code $\mathcal{C}$ forms the automorphism group $\operatorname{Aut}(\mathcal{C})$ of $\mathcal{C}$.

In the next section we investigate the possible types of automorphisms of order 21 of a binary doubly-even $[88,44,16]$ self-dual code. Further, we present a construction of a binary $[88,44,16]$ self-dual code having an automorphism of order 21 , and at last we list the new 35 codes. 
2. Automorphisms of order 21 of a binary doubly-even [88, 44, 16] self-dual code. Let $\mathcal{C}$ be a $[88,44,16]$ self-dual code having an automorphism $\sigma$ of order 21. Then, $\sigma$ is a permutation and, without loss of generality we may write

$$
\begin{aligned}
\sigma= & \Omega_{1} \Omega_{2} \ldots \Omega_{t_{1}} \Omega_{t_{1}+1} \Omega_{t_{1}+2} \ldots \Omega_{t_{1}+t_{2}} \\
& \Omega_{t_{1}+t_{2}+1} \Omega_{t_{1}+t_{2}+2} \ldots \Omega_{t_{1}+t_{2}+t_{3}} \\
& \Omega_{t_{1}+t_{2}+t_{3}+1} \Omega_{t_{1}+t_{2}+t_{3}+2} \ldots \Omega_{t_{1}+t_{2}+t_{3}+f}
\end{aligned}
$$

where $\Omega_{i}$ is a cycle of length 3 for $1 \leq i \leq t_{1}$, a cycle of length 7 for $t_{1}+1 \leq$ $i \leq t_{1}+t_{2}$, and a cycle of length 21 for $t_{1}+t_{2}+1 \leq i \leq t_{1}+t_{2}+t_{3}$. For $t_{1}+t_{2}+t_{3}+1 \leq i \leq t_{1}+t_{2}+t_{3}+f$ the symbol $\Omega_{i}$ represents a fixed point. For short we say that $\sigma$ is of type $21-\left(t_{1}, t_{2}, t_{3} ; f\right)$. From [3, Proposition 3.1] it follows that $\mathcal{C}$ has also automorphisms of type $3-\left(7 t_{3}+t_{1} ; 7 t_{2}+f\right)$ and type $7-\left(3 t_{3}+t_{2} ; 3 t_{1}+f\right)$. The possible automorphisms of order 3 and order 7 of an extremal self-dual binary code of length 88 are of types $3-(28 ; 4), 3-(26 ; 10)$, $3-(24 ; 16), 3-(22 ; 22), 3-(16 ; 40), 3-(14 ; 46), 7-(12 ; 4)$ and $7-(11 ; 11)$ [14, Theorem 1]. Hence, the type of the automorphism $\sigma$ can be $21-(0,0,4 ; 4)$, $21-(1,3,3 ; 1), 21-(0,6,2 ; 4), 21-(2,5,2 ; 5), 21-(0,5,2 ; 11), 21-(1,2,3 ; 8)$ and $21-(3,2,3 ; 2)$.

Similar to [3] we define

$$
F_{\sigma}(\mathcal{C})=\{v \in \mathcal{C} \mid v \sigma=v\}
$$

and

$$
\begin{aligned}
& E_{\sigma}(\mathcal{C})=\left\{v \in \mathcal{C} \mid w t\left(v \mid \Omega_{i}\right)\right. \equiv 0(\bmod 2), \\
&\left.i=1, \ldots, t_{1}+t_{2}+t_{3}+f\right\},
\end{aligned}
$$

where $v \mid \Omega_{i}$ is the restriction of $v$ to $\Omega_{i}$.

It is clear that $v \in F_{\sigma}(\mathcal{C})$, if and only if $v \in \mathcal{C}$ and the coordinates of $v$ are constant on each cycle $\Omega_{j}, j=1,2, \ldots, t_{1}+t_{2}+t_{3}+f$. The map $\pi$ is defined by

$$
\pi: F_{\sigma}(\mathcal{C}) \rightarrow \mathbb{F}_{2}^{t_{1}+t_{2}+t_{3}+f}, \quad \pi\left(v \mid \Omega_{i}\right)=v_{j},
$$

for some $j \in \Omega_{i}, i=1,2, \ldots, t_{1}+t_{2}+t_{3}+f$.

Let $\sigma$ be of type $21-(0,5,2 ; 11)$. Then, $\pi\left(F_{\sigma}(\mathcal{C})\right)$ is a binary self-dual $[18,9]$ code [3, Proposition 3.2]. 
Theorem 1 [10, Theorem 11]. Let $\mathcal{C}$ be a self-dual code of length $n=$ $n_{a}+n_{b}$ over $G F(q)$. Partition the generator matrix of $\mathcal{C}$ as follows:

$$
\begin{aligned}
& n_{a} n_{b} \\
& \begin{array}{ll}
k_{a} \\
k_{b} \\
k_{d}
\end{array} \quad\left(\begin{array}{cc}
A & O \\
O & B \\
D & E
\end{array}\right),
\end{aligned}
$$

where $k_{a}$ and $k_{b}$ are to be chosen as large as possible. Then

i) $k_{d}=\operatorname{rank} D=\operatorname{rank} E$,

ii) $k_{b}=1 / 2 n-\left(n_{a}-k_{a}\right)$,

iii) the code generated by the rows of $A$ and $D$ is the dual of the code generated by the rows of $A$.

Therefore, the generator matrix of any binary [18, 9] self-dual code can be presented in the form:

$$
\begin{array}{ll}
k_{a} \\
k_{b} \\
k_{d}
\end{array} \quad\left(\begin{array}{cc}
7 & 11 \\
A & O \\
O & B \\
D & E
\end{array}\right),
$$

where $k_{b}=2+k_{a}$. Then, $k_{b} \geq 2$. If a binary self-dual [18, 9] code generates $\pi\left(F_{\sigma}(\mathcal{C})\right)$, then the matrix $B$ generates $\left[11, k_{b}, d_{b}\right]$ code where $k_{b} \geq 2, d_{b} \geq 16$. So, the automorphism $\sigma$ is not of type $21-(0,5,2 ; 11)$.

In a similar way one can show that the automorphism $\sigma$ is not of type $21-(1,2,3 ; 8)$ either.

Therefore if an extremal binary $[88,44,16]$ self-dual doubly-even code has an automorphism of order 21 , then its type is $21-(0,0,4 ; 4), 21-(1,3,3 ; 1)$, $21-(0,6,2 ; 4), 21-(2,5,2 ; 5)$ or $21-(3,2,3 ; 2)$.

3. Construction of a Self-Dual $[88,44,16]$ code with an automorphism of type $21-(0,0,4 ; 4)$. Let now the permutation $\sigma$ of type $21-(0,0,4 ; 4)$ be an automorphism of $\mathcal{C} . F_{\sigma}(\mathcal{C})$ and $E_{\sigma}(\mathcal{C})$ are defined as in (4) and (5).

The next proposition follows from [8, Theorems 1-3].

Proposition 2. Let $\mathcal{C}$ be a self-dual doubly-even code of length 88 with an automorphism $\sigma$ of type $21-(0,0,4 ; 4)$. Then,

(1) $\mathcal{C}=F_{\sigma}(\mathcal{C}) \oplus E_{\sigma}(\mathcal{C})$. 
(2) $F_{\sigma}(\mathcal{C})$ and $E_{\sigma}(\mathcal{C})$ are $\sigma$-invariant, that is, invariant under the action of $\sigma$.

(3) The subcodes $F_{\sigma}(\mathcal{C})$ and $E_{\sigma}(\mathcal{C})$ have dimensions 4 and 44 respectively.

(4) $\pi\left(F_{\sigma}(\mathcal{C})\right)$ is a self-dual code of length 8.

The image $\pi\left(F_{\sigma}(\mathcal{C})\right)$ is a binary self-dual $[8,4]$ code. The only such codes are $C_{2}^{4}$ and $A_{8}$. Let $\pi\left(F_{\sigma}(\mathcal{C})\right)=A_{8}$. Then as a generator matrix of $F_{\sigma}(\mathcal{C})$ we can consider the following matrix:

$$
X=\left(\begin{array}{llll|llll}
\mathbf{1} & & & & & 1 & 1 & 1 \\
& \mathbf{1} & & & 1 & & 1 & 1 \\
& & \mathbf{1} & & 1 & 1 & & 1 \\
& & & \mathbf{1} & 1 & 1 & 1 &
\end{array}\right),
$$

where $\mathbf{1}$ is the all-one vector of length 21 and the blanks are zeroes.

Denote by $\mathcal{P}$ the set of even-weight polynomials in the factor-ring $\mathcal{R}_{21}=\mathbb{F}_{2}[x] /\left(x^{21}-1\right)$. The factorization of the polynomial $x^{21}-1$ over the binary field is given by $x^{21}-1=h_{0}(x) h_{1}(x) h_{2}(x) h_{3}(x) h_{4}(x) h_{5}(x)$, where

$h_{0}(x)=1+x, h_{1}(x)=1+x+x^{2}, h_{2}(x)=1+x+x^{3}, h_{3}(x)=1+x^{2}+x^{3}$, $h_{4}(x)=1+x+x^{2}+x^{4}+x^{6}$ and $h_{5}(x)=1+x^{2}+x^{3}+x^{4}+x^{5}+x^{6}$ are irreducible polynomials over $\mathbb{F}_{2}$.

Let $I_{j}$ be the ideal of $\mathcal{R}_{21}$ generated by the polynomial $\frac{x^{21}-1}{h_{j}(x)}$. Then $I_{j}$ is a cyclic code which is isomorphic to the field $\mathbb{F}_{2}^{\operatorname{deg} h_{j}(x)}$ for $j=1,2,3,4,5$ and, moreover, $\mathcal{P}=I_{1} \oplus I_{2} \oplus I_{3} \oplus I_{4} \oplus I_{5}$. The orthogonal idempotent of $I_{j}$, $j=1, \ldots, 5$ is $\epsilon_{j}(x)=e_{0}+e_{1} x+e_{2} x^{2}+\cdots+e_{20} x^{20}$, where $\epsilon_{j}$ are:

\begin{tabular}{c|c}
\hline$j$ & $e_{0} e_{1} \ldots e_{20}$ \\
\hline 1 & 011011011011011011011 \\
\hline 2 & 111010011101001110100 \\
\hline 3 & 100101110010111001011 \\
\hline 4 & 011010011001001010000 \\
\hline 5 & 000001010010011001011 \\
\hline
\end{tabular}

As a primitive element of $I_{j}, j=1, \ldots, 5$, we use $\mu_{j}(x)=m_{0}+m_{1} x+$ $m_{2} x^{2}+\cdots+m_{20} x^{20}$, where $\mu_{j}$ are:

\begin{tabular}{c|c}
\hline$j$ & $m_{0} m_{1} \quad \ldots \quad m_{20}$ \\
\hline 1 & 110110110110110110110 \\
\hline 2 & 100111010011101001110 \\
\hline 3 & 101110010111001011100 \\
\hline 4 & 011011110000101110101 \\
\hline 5 & 010101110100001111011 \\
\hline
\end{tabular}


Using GaP [17], the minimum distance of the cyclic codes $I_{1}, \ldots, I_{5}$ is calculated. We obtain that $I_{1}, I_{2}, I_{3}, I_{4}$ and $I_{5}$ are respectively $[21,2,14]$, [21, $3,12],[21,3,12],[21,6,8]$ and $[21,6,8]$ codes.

Let $E_{\sigma}(\mathcal{C})^{*}$ be the subcode $E_{\sigma}(\mathcal{C})$ with the last four coordinates deleted. We define the map $\varphi: E_{\sigma}(\mathcal{C})^{*} \rightarrow \mathcal{P}^{4}$ by identifying the restricted vector $v \mid \Omega_{i}=$ $\left(v_{0}, v_{1}, \ldots, v_{20}\right)$ with the polynomial $\varphi\left(v \mid \Omega_{i}\right)(x)=v_{0}+v_{1} x+\cdots+v_{20} x^{20}$ for $i=1,2,3,4$.

From [16, Lemma 6] $\varphi\left(E_{\sigma}(\mathcal{C})^{*}\right)$ is a self-orthogonal code in $\mathcal{P}^{4}$ under the inner product $\langle u, v\rangle=\sum_{i=1}^{4} u_{i}(x) v_{i}\left(x^{-1}\right)$. Therefore, we can take a generator matrix for $\varphi\left(E_{\sigma}(\mathcal{C})^{*}\right)$ of the form

$$
Y^{\prime}=\left(\begin{array}{cccc}
\epsilon_{1}(x) & 0 & \alpha_{1}(x) & \alpha_{2}(x) \\
0 & \epsilon_{1}(x) & \alpha_{3}(x) & \alpha_{4}(x) \\
\epsilon_{2}(x) & 0 & \beta_{1}(x) & \beta_{2}(x) \\
0 & \epsilon_{2}(x) & \beta_{3}(x) & \beta_{4}(x) \\
\beta_{1}\left(x^{-1}\right) & \beta_{3}\left(x^{-1}\right) & \epsilon_{3}(x) & 0 \\
\beta_{2}\left(x^{-1}\right) & \beta_{4}\left(x^{-1}\right) & 0 & \epsilon_{3}(x) \\
\epsilon_{4}(x) & 0 & \gamma_{1}(x) & \gamma_{2}(x) \\
0 & \epsilon_{4}(x) & \gamma_{3}(x) & \gamma_{4}(x) \\
\gamma_{1}\left(x^{-1}\right) & \gamma_{3}\left(x^{-1}\right) & \epsilon_{5}(x) & 0 \\
\gamma_{2}\left(x^{-1}\right) & \gamma_{4}\left(x^{-1}\right) & 0 & \epsilon_{5}(x)
\end{array}\right),
$$

where $\alpha_{i}(x) \in I_{1}$, for $i=1,2,3,4, \beta_{i}(x) \in I_{2}$ and $\beta_{i}\left(x^{-1}\right) \in I_{3}, i=1,2,3,4$, and $\gamma_{i}(x) \in I_{4}, \gamma_{i}\left(x^{-1}\right) \in I_{5}$ for $i=1,2,3,4$, whereas $\epsilon_{i}(x), i=1,2,3,4,5$ are defined above.

The corresponding generator matrix of the subcode $E_{\sigma}(\mathcal{C})^{*}$ is

$$
Y=\left(\begin{array}{rll}
y_{1,1} & \ldots & y_{1,4} \\
\vdots & \ddots & \vdots \\
y_{10,1} & \ldots & y_{10,4}
\end{array}\right),
$$

where $y_{i, j}, i=1,2, j=1, \ldots, 4$ are right-circulant $2 \times 21$ matrices, $y_{i, j}$ for $i=3, \ldots, 6, j=1, \ldots, 4$ are right-circulant $3 \times 21$ matrices, $y_{i, j}$ for $i=7, \ldots, 10$, $j=1, \ldots, 4$, are right-circulant $6 \times 21$ matrices. The first rows of the circulants correspond to the polynomials of the matrix $Y^{\prime}$. Thus, we constructed a possible generator matrix of $\mathcal{C}$.

Proposition 3. Let a binary self-dual doubly-even code $\mathcal{C}$ of length 88 have an automorphism $\sigma$ of type $21-(0,0,4 ; 4)$. Then a possible generator matrix 
of $\mathcal{C}$ can be written as

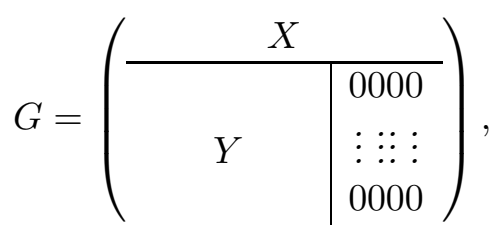

where $X$ and $Y$ are defined in (7) and (8).

A computer check shows that many self-dual doubly-even codes with a generator matrix of the type (9) are extremal. Here we present 35 examples $\mathcal{C}_{1}$, $\mathcal{C}_{2}, \ldots, \mathcal{C}_{35}$ of extremal codes. To define completely their generator matrices $G_{1}$, $\ldots, G_{35}$, it is sufficient to give the submatrix $Y$ of $G$ in (9). The matrix $Y$ is determined by the circulant matrices $y_{i, j}, i=1, \ldots, 12, j=1, \ldots, 6$ whose first rows are vectors corresponding to polynomials of the matrix $Y^{\prime}$. The values of the polynomials in $Y^{\prime}$ for the codes $\mathcal{C}_{1}, \mathcal{C}_{2}, \ldots, \mathcal{C}_{35}$ are as follows: $\alpha_{1}(x)=\alpha_{4}(x)=0$, $\alpha_{2}(x)=\alpha_{3}(x)=\mu_{1}(x) ; \beta_{1}(x)=0, \beta_{i}(x)$ is 0 or $\mu_{2}^{t_{i}}(x)$ for $i=2,3,4$ and $t_{i}=1, \ldots, 7 ; \gamma_{1}(x)=\epsilon_{4}(x), \gamma_{i}(x)$ is 0 or $\mu_{4}^{s_{i}}(x)$ for $i=2,3,4$ and $s_{i}=1, \ldots, 63$. The values of the degrees $t_{i}$ and $s_{i}$ for $i=2,3,4$ are listed in Table 1 . We note that if the value of $\gamma_{i}(x)$ or $\beta_{i}(x)$ is 0 , then the corresponding entry for $t_{i}$ or $s_{i}$ is empty.

The weight enumerator of an extremal doubly-even self-dual $[88,44,16]$ code is uniquely determined [4]:

$$
\begin{aligned}
W_{C}= & 1+32164 y^{16}+6992832 y^{20}+535731625 y^{24}+16623384448 y^{28}+ \\
& 225426781470 y^{32}+\cdots
\end{aligned}
$$

To prove the inequivalence of the codes we use the same invariants as in [6] and [5]. Let $M$ be the set of all 32164 codewords of weight 16 and $A_{i, j}$ be the number of the codewords of $M$ that have one at the coordinate positions $i$ and $j$. It is clear that the set of numbers $\left\{A_{i, j} \mid 1 \leq i<j \leq 88\right\}$ is an invariant for equivalent codes. So, the smallest and the largest element $m(2)$ and $M(2)$, respectively, in the set are invariants as well. Table 1 .

The values of $m(2)$ and $M(2)$ for the codes $\mathcal{C}_{1}, \mathcal{C}_{2}, \ldots, \mathcal{C}_{35}$ are listed in

Table 1 implies that the presented new 35 extremal self-dual codes of length 88 are inequivalent and, moreover, together with the data in [7] and [5] it follows that these codes and the codes given in [6] and [5] are inequivalent as well. 
Table 1. Matrices $Y^{\prime}$ and invariants

\begin{tabular}{c|c|c|c|c|c|c||c|c}
\hline Code & $t_{2}$ & $t_{3}$ & $t_{4}$ & $s_{2}$ & $s_{3}$ & $s_{4}$ & $M(2)$ & $m(2)$ \\
\hline $\mathcal{C}_{1}$ & 1 & 1 & 1 & 63 & 1 & 21 & 1071 & 672 \\
\hline $\mathcal{C}_{2}$ & 1 & 1 & 1 & 3 & 2 & 30 & 1080 & 819 \\
\hline $\mathcal{C}_{3}$ & 1 & 1 & 1 & 3 & 1 & 27 & 1089 & 777 \\
\hline $\mathcal{C}_{4}$ & 5 & 3 & 1 & & 1 & 28 & 1092 & 756 \\
\hline $\mathcal{C}_{5}$ & 7 & 1 & 1 & & 2 & 42 & 1095 & 714 \\
\hline $\mathcal{C}_{6}$ & 1 & 1 & 1 & & 1 & 54 & 1098 & 714 \\
\hline $\mathcal{C}_{7}$ & 1 & 1 & 1 & 3 & 2 & 39 & 1101 & 777 \\
\hline $\mathcal{C}_{8}$ & 1 & 1 & 1 & & 2 & 26 & 1104 & 777 \\
\hline $\mathcal{C}_{9}$ & 1 & 1 & 1 & 3 & 1 & 3 & 1107 & 801 \\
\hline $\mathcal{C}_{10}$ & 7 & 1 & 1 & & 2 & 19 & 1110 & 864 \\
\hline $\mathcal{C}_{11}$ & 7 & 1 & 1 & & 2 & 11 & 1113 & 738 \\
\hline $\mathcal{C}_{12}$ & 7 & 7 & 1 & & 1 & 26 & 1113 & 777 \\
\hline $\mathcal{C}_{13}$ & 1 & 1 & 1 & & 1 & 6 & 1116 & 672 \\
\hline $\mathcal{C}_{14}$ & 7 & 7 & 1 & & 1 & 25 & 1131 & 861 \\
\hline $\mathcal{C}_{15}$ & 1 & 1 & 1 & 3 & 1 & 20 & 1134 & 819 \\
\hline $\mathcal{C}_{16}$ & 1 & 1 & 1 & 3 & 1 & 45 & 1137 & 777 \\
\hline $\mathcal{C}_{17}$ & 1 & 1 & 1 & 1 & 1 & 5 & 1152 & 882 \\
\hline $\mathcal{C}_{18}$ & 1 & 1 & 1 & & 1 & 8 & 1155 & 630 \\
\hline $\mathcal{C}_{19}$ & 1 & 1 & 1 & & 1 & 21 & 1158 & 780 \\
\hline $\mathcal{C}_{20}$ & 7 & 1 & 1 & & 2 & 62 & 1176 & 630 \\
\hline $\mathcal{C}_{21}$ & 1 & 1 & 1 & & 1 & 52 & 1179 & 735 \\
\hline $\mathcal{C}_{22}$ & 1 & 1 & 1 & & 2 & 23 & 1197 & 693 \\
\hline $\mathcal{C}_{23}$ & 7 & 1 & 1 & & 2 & 61 & 1218 & 717 \\
\hline $\mathcal{C}_{24}$ & 7 & 7 & 1 & & 1 & 12 & 1221 & 903 \\
\hline $\mathcal{C}_{25}$ & 1 & 1 & 1 & & 2 & 30 & 1239 & 693 \\
\hline $\mathcal{C}_{26}$ & 7 & 1 & 1 & & 3 & 1 & 1242 & 840 \\
\hline $\mathcal{C}_{27}$ & 7 & 1 & 1 & & 2 & 59 & 1263 & 885 \\
\hline $\mathcal{C}_{28}$ & 7 & 1 & 1 & & 2 & 21 & 1281 & 735 \\
\hline $\mathcal{C}_{29}$ & 5 & 3 & 1 & & 1 & 12 & 1302 & 756 \\
\hline $\mathcal{C}_{30}$ & 1 & 1 & 1 & & 1 & 55 & 1323 & 612 \\
\hline $\mathcal{C}_{31}$ & 7 & 1 & 1 & & 2 & 57 & 1344 & 843 \\
\hline $\mathcal{C}_{32}$ & 1 & 1 & 1 & 63 & 1 & 37 & 1347 & 798 \\
\hline $\mathcal{C}_{33}$ & 1 & 1 & 1 & 3 & 1 & 21 & 1365 & 840 \\
\hline $\mathcal{C}_{34}$ & 7 & 1 & 1 & & 3 & 8 & 1368 & 840 \\
\hline $\mathcal{C}_{35}$ & 1 & 1 & 1 & 3 & 1 & 6 & 1389 & 861 \\
\hline
\end{tabular}


Theorem 4. Up to equivalence there are at least 105 binary extremal self-dual doubly-even codes of length 88, where 35 are new.

Acknowledgment. The authors would like to thank the anonymous referees for their useful comments.

\section{REFERENCES}

[1] Conway J. H., V. Pless. On the enumeration of self-dual codes. J. Combin. Theory, ser. A, 28 (1980), 26-53.

[2] Conway J. H., V. Pless, N. J. Slonne. The binary self-dual codes of length up to 32: a revised enumeration. J. Combin. Theory, ser. A, 60 (1992), 183-195.

[3] Dontcheva R., A. J. van Zanten, S. M. Dodunekov. Binary self-dual codes with automorphisms of composite order. IEEE Trans. Inform. Theory, 50 (2004), 311-318.

[4] Dougherty S. T., T. A. Gulliver, M. Harada. Extremal binary selfdual codes. IEEE Trans. Inform. Theory, 43 (1997), 2036-2047.

[5] Goodwin V., V. Yorgov. New extremal self-dual doubly-even binary codes of length 88. Finite Fields and Applications, 11 (2005), 1-5.

[6] Gulliver T. A., M. Harada, J-L. Kim. Construction of new extremal self-dual codes. Discrete. Mathematics, 263 (2003), 81-91.

[7] Harada M. Private correspondence.

[8] Huffman W. C. Decomposing and shortening codes using automorphisms. IEEE Trans. Inform. Theory, 32 (1986), 833-836.

[9] MacWilliams F. J., N. J. A. Sloane. The Theory of Error-Correcting Codes. Amsterdam: North-Holland, 1977.

[10] Pless V., N. J. A. Sloane, H. N. Ward. Ternary codes of minimum weight 6 and the classification of the self-dual codes of length 20. IEEE Trans. Inform. Theory, IT-26 (1980), 305-316.

[11] Pless V. A classification of self-orthogonal codes over GF(2). Discrete Mathematics, 3 (1972), 209-246. 
[12] Pless V., N. J. A. Slonne. On the Classification and Enumeration of Self-Dual Codes. J.Combinatorial Theory, 18 (1975), 313-335.

[13] RAins E. M. Shadow bounds for self-dual codes. IEEE Trans. Inform. Theory, 44 (1998), 134-139.

[14] Yorgov V. Y. Binary self-dual codes with automorphisms of odd order (in Russian). Problemi Peredachi Informatcii, 19 (1983), 11-24, English translation in Probl. Inform. Transm. 19 (1983), 260-270.

[15] Yorgov V. Y. On the extremal binary codes of length 32. In: Proceedings of the 4th Joint Swedish-Russian International Workshop on Information Theory, Gotland, Sweden, 1989, 275-279.

[16] Yorgova R. On binary self-dual codes with automorphisms. IEEE Trans. Inform. Theory, 54 (2008), 3345-3351.

[17] www.gap-system.org.

Radinka Yorgova

CIPR, University of Bergen, Norway

e-mail: radinka.yorgova@cipr.uib.no

Nuray At

Dept. of Electrical and Electronics Engineering

Anadolu University, Turkey

Received April 2, 2009

e-mail: nat@anadolu.edu.tr

Final Accepted June 11, 2009 